Filomat 31:9 (2017), 2713-2726

https://doi.org/10.2298/FIL1709713K

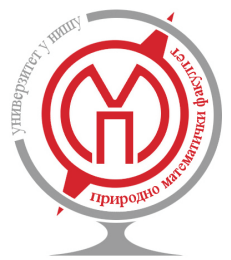

Published by Faculty of Sciences and Mathematics, University of Niš, Serbia

Available at: http://www.pmf.ni.ac.rs/filomat

\title{
An Efficient Iterative Algorithm for Solving Non-Linear Oscillation Problems
}

\author{
S.Ö. Korkut Uysal ${ }^{a}$, G. Tanoğlu ${ }^{b}$ \\ ${ }^{a}$ Izmir Kâtip Çelebi University, Balatçık Campus,Çiğli, Izmir,35620, Turkey \\ ${ }^{b}$ Izmir Institute of Technology, Gulbahce Campus, Urla, Izmir,35430, Turkey
}

\begin{abstract}
A new iterative method is presented for numerical solution of nonlinear evolutionary problems. The convergence properties of the proposed method are analysed in abstract framework by using the concepts of consistency, stability and order. Both the $\varphi$-functions and semigroup properties are used to overcome the presence of unboundedness of the operator. In order to confirm the theoretical results, the method is applied to three benchmark problems from the literature. The numerical results are compared with traditional splitting methods and confirm that the proposed method is more accurate as well as more efficient than the traditional splitting methods.
\end{abstract}

\section{Introduction}

Several phenomena in engineering and science exhibit substantially nonlinear behaviour. These phenomena are often modelled with the help of nonlinear differential equations. Due to non-availability of analytic solution for these nonlinear differential equations, researchers have developed several numerical methods to find approximate solutions of such equations. Operator splitting is one of the widely used technique in the literature. The basic idea of the operator splitting procedures is that instead of the sum, the operators are considered separately. This divides the original problem into sub-problems and the numerical solution of the original problem is obtained from numerical solutions of these sub-problems. The operator splitting techniques have been applied by many researchers to solve various types of differential equations numerically, see $[1,3,5,11,15]$ for linear non-autonomous case and $[13,16]$ for nonlinear differential equation.

There are certain disadvantages of the traditional splitting procedures and due to this reason a new type of operating splitting method called iterative splitting method was developed by Faragó and Geiser [7]. Further applications of this method can be seen in $[8,9]$. The iterative splitting method was also applied in combination with Newton's method to nonlinear differential equations in [10].

We proposed a new iterative splitting method and analysed its convergence properties for solving linear time-dependent differential equations in [18]. The present work is an extension of our earlier work [18] to nonlinear problems. In the present study, the essential tools in the construction of this new scheme include the iterative splitting process and the Magnus expansion.

2010 Mathematics Subject Classification. 65L05; 65L20; 65L70

Keywords. Non-linear Magnus Integrator; Exponential Integrator; Iterative Splitting; Non-linear Oscillation Problems ; Convergence Analysis.

Received: 10 August 2015; Accepted: 19 December 2015

Communicated by Maria Alessandra RAGUSA

Email addresses: silaovgu@gmail.com (S.Ö. Korkut Uysal), gamzetanoglu@iyte. edu.tr (G. Tanoğlu) 
In this work, we will develop as well as analyse a new splitting method for nonlinear evaluation equation of the following form:

$$
\frac{d u}{d t}=A(t, u) u(t), \quad 0 \leq t \leq t_{\text {end }},
$$

subject to the initial condition

$$
u(0)=u_{0} \in X,
$$

on some Banach space $X$, where $A(t, u)$ is an unbounded nonlinear operator. Here, the structure of the operator $A(t, u)$ suggests a decomposition into two parts in the following form:

$$
A(t, u(t)) u(t)=T u(t)+V(t, u(t)) u(t), \quad u(t): \mathbb{D}(T) \cap \mathbb{D}(V) \rightarrow X,
$$

with the unbounded operator $T: \mathbb{D}(T) \subset X \rightarrow X$ and bounded operator $V(t, u): \mathbb{D}(V) \in\left[0, t_{\text {end }}\right] \times X \rightarrow X$. Thus, we have

$$
\begin{aligned}
\dot{\omega}(t) & =T \omega(t)+V(t, v) v(t) \\
\omega(0) & =u_{0},
\end{aligned}
$$

and

$$
\begin{aligned}
\dot{v}(t) & =T \omega(t)+V(t, v) v(t) \\
v(0) & =u_{0},
\end{aligned}
$$

where $u(t)=v(t)$. Eq. (5) represents a homogeneous equation, however, due to the time-dependence and nonlinearity, we can not use the exponential function as a fundamental solution of this equation. We use Magnus expansion instead and with the help of the nonlinear Magnus expansion the fundamental solution of Eq. (5) can be expressed as

$$
u(t)=\exp \left(\Omega_{V}\left(t, u_{0}\right)\right) u_{0}
$$

where

$$
\begin{aligned}
\Omega^{[0]} & \equiv 0, \\
\Omega^{[1]} & =\int_{0}^{t} V\left(s, u_{0}\right) d s, \\
\Omega^{[m]} & =\sum_{k=0}^{m-2} \frac{B_{k}}{k !} \int_{0}^{t} a d_{\Omega^{[m-1]}}^{k} V\left(s, e^{\Omega^{[m-1]}} u_{0}\right) d s, \quad m \geq 2,
\end{aligned}
$$

and we assume the approximation $\Omega(t) \approx \Omega^{[m]}(t)$. The first order Magnus operator is obtained using Euler's formula and is given as follows:

$$
\Omega^{[1]}=\int_{0}^{t} V\left(s, u_{0}\right) d s=h V\left(0, u_{0}\right)+O\left(h^{2}\right) .
$$

The second order Magnus operator is obtained with the help of trapezoidal rule, and is given below:

$$
\begin{aligned}
\Omega^{[2]} & =\int_{0}^{h} V\left(s, e^{\Omega^{[1]}} u_{0}\right) d s \\
& =\frac{h}{2}\left(V\left(0, u_{0}\right)+V\left(h, e^{\Omega^{[1]}} u_{0}\right)\right)+O\left(h^{3}\right) .
\end{aligned}
$$

Detailed discussions about the nonlinear Magnus expansion can be found in the reference [4].

The structure of this paper is as follows: In Section 2, the algorithms of the new scheme are presented. In Section 3, convergence properties of the constructed scheme are studied in an abstract framework and in order to accomplish this, we use both the exponential integrator and the semigroup approaches for our analysis. In Section 4, several numerical examples are illustrated to confirm our theoretical results and efficiency of the given scheme. Finally, some conclusions are drawn in Section 5. 


\section{Proposed Iterative Splitting Method}

In this section, we will first give a general idea of splitting methods with a brief discussion of some of the well-known splitting methods. Then, we will derive an algorithm for the new iterative splitting scheme.

The general idea of splitting methods is introduced by considering the initial value problem given in Eq. (1) with the initial condition given in Eq. (2) on the time interval $\left[0, t_{\text {end }}\right]$ where $t_{\text {end }} \in \mathbb{R}^{+}$. As mentioned before in the introduction section, we suppose for $A(t, u)$ a two-term splitting of the form $T(u)+V(t, u)$.

Let us divide the integration interval $\left[0, t_{\text {end }}\right]$ in $n$ equal parts by the points $t_{0}, t_{1}, \ldots, t_{n}$, where the length of each subinterval is $h=t_{j+1}-t_{j}=t_{\text {end }} / n, j=0,1 \ldots n$. In the sequel, we give the formulas of splitting methods with the help of Magnus operator which was introduced earlier. For simplicity of the expression, we give the numerical solutions on $[0, h]$.

\subsection{Strang splitting}

We first deal with the well known splitting method, Strang splitting, and the solution is given as follows:

$$
U_{s p}(h)=e^{T \frac{h}{2}} e^{\frac{h}{2}\left(V\left(0, u_{0}\right)+V\left(h, e^{T \frac{h}{2}}\right)\right)} e^{T \frac{h}{2}} u_{0} .
$$

\subsection{Symmetrically weighted splitting}

Besides Strang splitting, there is another well-known second order splitting method in literature that is called symmetrically weighted splitting. The solution in this case is given below.

$$
U_{s p}(h)=\frac{1}{2}\left(e^{T h} e^{\frac{h}{2}\left(V\left(0, u_{0}\right)+V(h, u(h))\right)}+e^{\frac{h}{2}\left(V\left(0, u_{0}\right)+V(h, u(h))\right)} e^{T h}\right) u_{0} .
$$

Repeat this procedure, by taking $u_{0}=U(h)$ where $U$ denotes the numerical solution, until desired time is achieved.

\subsection{Derivation of the Algorithm for New Iterative Splitting Scheme}

In this section, we extend the scheme in [18] to nonlinear case. For this purpose, we apply the second order iterative process on each subinterval $\left[t_{j}, t_{j+1}\right]$ and is described below.

The sub-equations obtained from the original equations are given by

$$
\begin{array}{ll}
\dot{u}_{1}=T u_{1}+V\left(t, U\left(t_{j}\right)\right) U\left(t_{j}\right) & u_{1}\left(t_{j}\right)=U\left(t_{j}\right), \\
\dot{u}_{2}=T u_{1}+V\left(t, u_{2}\left(t_{j}\right)\right) u_{2}\left(t_{j}\right) & u_{2}\left(t_{j}\right)=U\left(t_{j}\right),
\end{array}
$$

where $u_{2}\left(t_{j}\right)=U\left(t_{j}\right)$ denotes the numerical approximation to the exact solution $u\left(t_{j}\right)$ at the time $t=t_{j}$ and $U\left(t_{0}\right)=u_{0}$. The formal solution of the sub-equations given in Eq. (12) and Eq. (13) on the time interval $[t, t+h]$ can be written as

$$
u_{i}(t+h)=\Phi_{i}(t+h, t) U(t)+\int_{t}^{t+h} \Phi_{i}(t+h, s) F_{i}(s) d s, \quad i=1,2,
$$

where $F_{1}=V(t, U(t)) U(t), F_{2}=T u_{1}(t)$ and

$$
\begin{aligned}
& \Phi_{1}(t+h, t)=e^{h T} \\
& \Phi_{2}(t+h, t)=e^{\frac{h}{2}\left[V(t, u(t))+V\left(t+h, u_{1}(t+h)\right)\right]} .
\end{aligned}
$$

Here $\Phi_{2}$ is obtained by means of nonlinear Magnus operator. Next we use the trapezoidal rule to approximate the integral:

$$
\int_{t}^{t+h} \Phi_{i} F_{i} d s=\frac{h}{2}\left[F_{i}(t+h)+\Phi_{i}(t+h, t) F_{i}(t)\right]+O\left(h^{3}\right) .
$$


Note that $\Phi_{i}(t+h, t+h)=I$ where $I$ is the identity operator. After combining approximation given in Eq. (14) with the iterative schemes given in Eqs. (12)-(13) and rearranging expressions, we obtain the first order approximation

$$
u_{1}^{n+1}=e^{T h}\left[u_{1}^{n}+\frac{h}{2} V\left(t_{n}, u_{0}\left(t_{n}\right)\right) u_{0}^{n}\right]+\frac{h}{2} V\left(t_{n}+h, u_{0}^{n+1}\right) u_{0}^{n+1},
$$

and the second order approximation

$$
u_{2}^{n+1}=e^{\frac{h}{2}\left[V\left(t_{n}, u^{n}\right)+V\left(t_{n}+h, u_{1}^{n+1}\right)\right]}\left[U\left(t_{n}\right)+\frac{h}{2} T u_{1}^{n}\right]+\frac{h}{2} T u_{1}^{n+1},
$$

where $U\left(t_{n}+h\right)=u_{2}\left(t_{n}+h\right)$ and $u_{i}^{n}=u_{i}\left(t_{n}\right)$. Repeat this procedure by taking $u_{0}\left(t_{n}\right)=u_{2}\left(t_{n}+h\right)$ for next interval until the desired time $t_{\text {end }}$ is reached.

\section{Convergence Analysis}

The main idea of this section is to analyze the convergence behavior of the method given in the previous section. For the convergence, we will investigate consistency and stability issues. We assume that $T$ is an unbounded operator whereas $V(t, u)$ is a bounded operator. In our analysis, we define an operator norm as $\|.\|_{X}$ on $\left(X,\|.\|_{X}\right)$. To simplify the notations, we will write $\|$.$\| instead of \|.\|_{X}$. Throughout this section $u(h)$ and $U(h)$ represent exact and numerical solutions, respectively.

In our proofs, we will use the following auxiliary assumptions:

Assumption 3.1. Let $X$ be the Banach space with the norm \|.\|. We assume $T$ is a linear operator on $X$ and that $T$ is the infinitesimal generates $C_{0}$ semigroup $e^{t T}$ on $X$. Particularly, this assumption implies that there exist constants $C$ and $\omega$ such that

$$
\left\|e^{t T}\right\| \leq C e^{\omega t} \leq 1, \quad t \geq 0
$$

Assumption 3.2. In order to simplify the calculations, we denote $V(t, u()) u.($.$) as g(u()$.$) throughout this$ section. Then, there exists a constant $\alpha_{k}, k=0,1,2, \ldots$ such that

$$
\left\|g^{(k)}(u(t))\right\| \leq \alpha_{k}, \quad k=0,1,2, \ldots
$$

Assumption 3.3. Let $V(t, u) \in \mathcal{D}(T) \cap H^{1}$. Then, there exists a constant $\tilde{S}_{k}, k=0,1,2, \ldots$ such that

$$
\left\|T g^{(k)}(u(t))\right\| \leq \tilde{S}_{k} u_{0}, \quad k=0,1,2, \ldots
$$

Assumption 3.4. Let $X$ be a Banach space with norm $\|$.$\| . Then, there exists a constant M_{k}, k=0,1,2, \ldots$ such that

$$
\left\|T u^{(k)}\right\| \leq M_{k} u_{0}, \quad k=0,1,2, \ldots
$$

Proposition 3.1. $\left[T, \varphi_{k}(s T)\right]=0, k=1,2,3, \ldots$ where $\left[T, e^{s T}\right]=0$.

Proof. This proof follows the line of induction. Suppose $k=1$. By using the definition of the $\varphi-$ function 
and the identity $\left[T, e^{s T}\right]=0$, we obtain

$$
\begin{aligned}
{\left[T, \varphi_{1}(s T)\right] } & =T \varphi_{1}(s T)-\varphi_{1}(s T) T \\
& =T \frac{e^{s T}-I}{s T}-\frac{e^{s T}-I}{s T} T \\
& =\frac{T e^{s T}-T}{s T}-\frac{e^{s T} T-T}{s T} \\
& =\frac{T e^{s T}-e^{s T} T}{s T} \\
& =\frac{\left[T, e^{s T}\right]}{s T} \\
& =0 .
\end{aligned}
$$

Next assume that $\left[T, \varphi_{k}(s T)\right]=0$. In order to show that $\left[T, \varphi_{k+1}\right]=0$, we note that the recurrence relation

$$
\varphi_{k+1}(s T)=\frac{\varphi_{k}(s T)-\varphi_{k}(0)}{s T}, \varphi_{0}(s T)=e^{s T}
$$

holds. This relation leads to

$$
\begin{aligned}
{\left[T, \varphi_{k+1}(s T)\right] } & =T \varphi_{k+1}(s T)-\varphi_{k+1}(s T) T \\
& =T \frac{\varphi_{k}(s T)-\varphi_{k}(0)}{s T}-\frac{\varphi_{k}(s T)-\varphi_{k}(0)}{s T} T \\
& =\frac{T \varphi_{k}(s T)-T \varphi_{k}(0)}{s T}-\frac{\varphi_{k}(s T) T-\varphi_{k}(0) T}{s T} \\
& =\frac{T \varphi_{k}(s T)-T \varphi_{k}(0)-\left(\varphi_{k}(s T) T-\varphi_{k}(0) T\right)}{s T} \\
& =\frac{T \varphi_{k}(s T)-\varphi_{k}(s T) T-\left(T \varphi_{k}(0)-\varphi_{k}(0) T\right)}{s T} \\
& =\frac{\left[T, \varphi_{k}(s T)\right]-\left[T, \varphi_{k}(0)\right]}{s T} .
\end{aligned}
$$

Due to the assumption $\left[T, \varphi_{k}(s T)\right]=0$ and the definition of $\varphi_{k}(0)$, we have

$$
\begin{aligned}
{\left[T, \varphi_{k+1}(s T)\right] } & =\frac{\left[T, \varphi_{k}(s T)\right]-\left[T, \varphi_{k}(0)\right]}{s T} \\
& =0 .
\end{aligned}
$$

Our general reference on the exponential integrator is the survey of Hochbruck and Ostermann [12]. Moreover, we refer to [6] for the exponential integrators in a framework of semigroup.

\subsection{Consistency Analysis}

Proposition 3.2. The proposed iterative splitting is first order if we consider (12) with the error bound

$$
\|u(h)-U(h)\| \leq \beta h^{2} .
$$

Here $\beta$ depends only on $\alpha_{1}$.

Proof. We define the local error by $e_{j}=U\left(t_{j}\right)-u\left(t_{j}\right), j=0,1,2, \ldots, n$. For simplicity we consider the time interval $[0, \mathrm{~h}]$ only. The exact solution of Eq. (1) can be written as

$$
u(h)=e^{T h} u_{0}+\int_{0}^{h} e^{T(h-s)} g(u(s)) d s .
$$


In order to establish the local error, the primary tool is to employ the variation-of-constant formula. Thus, the first order numerical solution of Eq. (1) is given by

$$
U(h)=e^{T h} u_{0}+\int_{0}^{h} e^{T(h-s)} g\left(u_{0}\right) d s .
$$

By subtracting Eq. (27) from Eq. (26) we obtain

$$
\begin{aligned}
\|u(h)-U(h)\| & =\left\|\int_{0}^{h} e^{T(h-s)} g(u(s))-g\left(u_{0}\right) d s\right\| \\
& \leq\left\|\int_{0}^{h} e^{T(h-s)}\left(\int_{0}^{s} \partial g(u(\rho)) d \rho\right) d s\right\| .
\end{aligned}
$$

Applying Assumption 3.1 and Assumption 3.2, we get

$$
\|u(h)-U(h)\| \leq h^{2} \beta .
$$

Proposition 3.3. Let Assumption 3.1 and Assumption 3.3 be fulfilled. Then, the proposed method has second order approximation if we consider (16) with the error bound

$$
\|u(h)-U(h)\| \leq G h^{3}
$$

Here $G$ depends on $E_{1}, \tilde{R}_{2}$ and $\tilde{S}_{1}$ where $E_{1}$ is the bound for $\left\|\Phi_{2}(h, s)\right\|$ and $\tilde{R}_{2}$ is the bound for $\varphi_{2}(h T)$.

Proof. A primary tool for the derivation of our local error representation is variation-of-constant formula. The fundamental sets of solutions of Eqs. (12) and (13), respectively, are given as follows:

$$
\begin{aligned}
& u_{1}(h)=e^{T h} u_{0}+\int_{0}^{h} e^{T(h-s)} V\left(u_{0}\right) u_{0} d s, \\
& u_{2}(h)=\Phi_{2}(h, 0) u_{0}+\int_{0}^{h} \Phi_{2}(h, s) T u_{1}(s) d s .
\end{aligned}
$$

Here, $\Phi_{2}(h, 0)$ is the numerical flow of Eq. (13). Especially, we construct this flow by using Magnus expansion due to the nonlinearity of the operator.

On the other hand, the exact solution can be written as in the following forms:

$$
u(h)=e^{T h} u_{0}+\int_{0}^{h} e^{T(h-s)} V(u(s)) u(s) d s,
$$

or

$$
u(h)=\Phi_{2}(h, 0) u_{0}+\int_{0}^{h} \Phi_{2}(h, s) T u(s) d s .
$$

Moreover,

$$
u(h)=u_{0}+\int_{0}^{h} F(u(s)) d s,
$$

where $F(u(s))=T u(s)+V(s, u(s)) u(s)$.

By substituting Eq. (31) into Eq. (33), we have

$$
u_{2}(h)=\Phi_{2}(h, 0) u_{0}+\int_{0}^{h} \Phi_{2}(h, s) T e^{T h} u_{0} d s+\int_{0}^{h} \Phi_{2}(h, s) T\left(\int_{0}^{s} e^{T(s-\rho)} V\left(u_{0}\right) u_{0} d \rho\right) d s .
$$


As we mentioned before, set $V(t, u) u$ as $g(u(t))$. Then,

$$
u(h)=\Phi_{2}(h, 0) u_{0}+\int_{0}^{h} \Phi_{2}(h, s) T e^{T h} u_{0} d s+\int_{0}^{h} \Phi_{2}(h, s) T\left(\int_{0}^{s} e^{T(s-\rho)} g(u(\rho)) d \rho\right) d s .
$$

Since $T$ is unbounded, to prove the consistency of the second order method, we restrict ourselves to the $\varphi-$ function representation. Then, Eq. (35) and Eq. (36) can be represented by using $\varphi-$ function and we have the following:

$$
u_{2}(h)=\Phi_{2}(h, 0) u_{0}+\int_{0}^{h} \Phi_{2}(h, s) T e^{T h} u_{0} d s+\int_{0}^{h} \Phi_{2}(h, s) T \varphi_{1}(s T) s g\left(u_{0}\right) d s,
$$

whereas the exact one is

$$
\begin{aligned}
u(h) & =\Phi_{2}(h, 0) u_{0}+\int_{0}^{h} \Phi_{2}(h, s) T e^{T h} u_{0} d s+\int_{0}^{h} \Phi_{2}(h, s) T s\left(\sum_{k=1}^{p} \varphi_{k}(s T) s^{k-1} g^{(k-1)}\left(u_{0}\right)\right) d s \\
& +\int_{0}^{h} \Phi_{2}(h, s) \int_{0}^{\tau} \frac{(\tau-\xi)^{p-1}}{(p-1) !} T g^{(p)}(\xi) d \xi d s .
\end{aligned}
$$

For estimating the error bound, we subtract Eq. (37) from Eq. (38) and obtain

$$
u(h)-u_{2}(h)=\int_{0}^{h} \Phi_{2}(h, s) T s\left(\sum_{k=2}^{p} \varphi_{k}(s T) s^{k-1} g^{(k-1)}\left(u_{0}\right)\right) d s .
$$

Here, the auxiliary proposition, that is, Proposition 3.1 is used. This yields

$$
u(h)-u_{2}(h)=\int_{0}^{h} \Phi_{2}(h, s) s\left(\sum_{k=2}^{p} \varphi_{k}(s T) s^{k-1} T g^{(k-1)}\left(u_{0}\right)\right) d s .
$$

Thus,

$$
\left\|u(h)-u_{2}(h)\right\|=\left\|\int_{0}^{h} \Phi_{2}(h, s)\left(\sum_{k=2}^{p} \varphi_{k}(s T) s^{k} T g^{(k-1)}\left(u_{0}\right)\right) d s\right\|+\int_{0}^{h} \Phi_{2}(h, s) \int_{0}^{\tau} \frac{(\tau-\xi)^{p-1}}{(p-1) !} T g^{(p)}(\xi) d \xi d s .
$$

Hence,

$$
\begin{aligned}
\left\|u(h)-u_{2}(h)\right\| & \leq \int_{0}^{h}\left\|\Phi_{2}(h, s) s^{2} \varphi_{2}(s T) T g^{\prime}\left(u_{0}\right)\right\| d s+\delta_{3}^{p} \\
& \leq E_{1} \int_{0}^{h} s^{2}\left\|\varphi_{2}(s T) T g^{\prime}\left(u_{0}\right)\right\| d s+O\left(h^{4}\right) .
\end{aligned}
$$

Here, by means of the boundedness of $V(t, u)$ operator, $E_{1}$ denotes the bound for $\left\|\Phi_{2}(h, s)\right\|$. Moreover, the remainder term $\delta_{3}^{p}$ is bounded by $O\left(h^{4}\right)$.

Using Assumption 3.1 and the following definition of $\varphi$ operators

$$
\varphi_{k}(h T)=\int_{0}^{1} e^{h(1-\theta) T} \frac{\theta^{k-1}}{(k-1) !} d \theta, \quad k=1,2,3, \ldots
$$

we can deduce that

$$
\begin{aligned}
&\left\|\varphi_{k}(h T)\right\| \leq \int_{0}^{1}\left\|\frac{\theta^{k-1}}{(k-1) !}\right\| d \theta . \\
&\left\|\varphi_{k}(h T)\right\| \leq \tilde{R}_{k}, \quad k=1,2,3, \ldots
\end{aligned}
$$


Using Eq. (41) and Assumption 3.3, we obtain

$$
\left\|u(h)-u_{2}(h)\right\| \leq h^{3} E_{1} \tilde{R}_{2} \tilde{S}_{1}+O\left(h^{4}\right) .
$$

Here $\tilde{R}_{2}$ is the bound for $\varphi_{2}(h T)$. It proves that the second order method is also consistent.

\subsection{Stability Analysis}

Proposition 3.4. The new first order iterative splitting scheme is stable on $\left[0, t_{\text {end }}\right]$ with the bound

$$
\left\|U^{n}\right\| \leq K_{1}\left\|u_{0}\right\|
$$

where the constant $K_{1}$ depends on $t_{\text {end }}$ and $\alpha_{0}$.

Proof. To prove the stability of the new first order method, we employ the standard techniques. For this purpose, we start with rewriting the formulation of the method as in the following form

$$
U^{1}=U(h)=e^{T h} u_{0}+G_{1}, U^{0}=u_{0},
$$

where

$$
G_{1}=\int_{0}^{h} e^{T(h-s)} V\left(s, u_{0}\right) u_{0} d s,
$$

which is bounded by $\left\|G_{1}\right\| \leq h \alpha_{0}\left\|u_{0}\right\|$. Rearranging Eq. (44), we get

$$
\begin{aligned}
&\left\|U^{1}\right\|=\left\|e^{T h} U^{0}+G_{1}\right\| \leq\left\|e^{T h} U^{0}\right\|+\left\|G_{1}\right\|, \\
&\left\|U^{1}\right\| \leq\left\|u_{0}\right\|+h \alpha_{0}\left\|u_{0}\right\|=\left(1+h \alpha_{0}\right)\left\|u_{0}\right\| .
\end{aligned}
$$

Recursively we get the stability polynomial for iterative scheme at first order,

$$
\left\|U^{n}\right\| \leq\left(1+h \alpha_{0}\right)^{n}\left\|u_{0}\right\| \leq e^{t_{\text {end }} \alpha_{0}}\left\|u_{0}\right\| .
$$

Thus, the proof is concluded.

Proposition 3.5. Similarly, the new second order iterative splitting scheme is stable if $\|\Phi(t, s)\| \leq 1$ on $\left[0, t_{\text {end }}\right]$ with the bound

$$
\left\|U^{n}\right\| \leq K_{2} u_{0}
$$

where $K_{2}$ depends on $E_{1}, t_{\text {end }}, \tilde{R}_{1}, \tilde{S}_{0}$ and $M_{0}$. The constants $E_{1}$ and $R_{1}$ are the bounds for $\Phi_{2}(h, s)$ and $\varphi_{1}$, respectively.

Proof. The proof follows the line of previous one. In order to obtain the stability bound for the second order method, Eq. (13) is rewritten as in the following form

$$
U^{1}=U(h)=\Phi_{2}(t+h, t) U^{0}+\int_{0}^{h} \Phi_{2}(h, s) T u_{1} d s,
$$

where $U^{0}=u(0)$. Substituting $u_{1}$ into Eq. (47) and using the linearity of $T$ leads to

$$
U^{1}=U(h)=\Phi_{2}(h, 0) u_{0}+\int_{0}^{h} \Phi_{2}(h, s) T e^{T h} u_{0} d s+\int_{0}^{h} \Phi_{2}(h, s) T\left(\int_{0}^{s} e^{T(s-\rho)} g\left(u_{0}\right) d \rho\right) d s .
$$

Rearranging by means of the $\varphi$-functions, we have

$$
U^{1}=U(h)=\Phi_{2}(h, 0) u_{0}+\int_{0}^{h} \Phi_{2}(h, s) T e^{T h} u_{0} d s+\int_{0}^{h} \Phi_{2}(h, s) T s \varphi_{1}(s T) g\left(u_{0}\right) d s .
$$

After taking norm, Assumption 3.3 and boundedness of $\Phi_{2}(h, s)$ lead to

$$
\|U\|^{1} \leq E_{1} u_{0}+\int_{0}^{h} E_{1} M_{0} u_{0} d s+\int_{0}^{h} E_{1} s \phi_{1}(s T)\left\|T g\left(u_{0}\right)\right\| d s .
$$


Using Assumption 3.2., we have

$$
\begin{aligned}
\|U\|^{1} & \leq E_{1} u_{0}+h E_{1} M_{0} u_{0}+\frac{h^{2}}{2} E_{1} \tilde{R}_{1} U_{0} \\
& \leq E_{1}\left(1+h M_{0}+h^{2} \frac{\tilde{R}_{1}}{2}\right) U_{0}
\end{aligned}
$$

where $\tilde{R}_{1}$ is the bound for $\varphi_{1}(s T)$ as shown in Eq. (41). Recursively,

$$
\|U\|^{n} \leq E_{1}^{n} e^{t_{\text {end }} \gamma} U_{0}
$$

where $\gamma$ depends on $\tilde{R}_{1}$ and $M_{0}$. As a consequence, the second order scheme is stable if $\left\|\Phi_{2}\right\| \leq 1$.

Proposition 3.6. The global error of the new iterative splitting at the second order is bounded by

$$
\left\|U^{n}(h)-u^{n}(h)\right\| \leq J_{1} h^{2}+O\left(h^{3}\right)
$$

Proof. We use the following insignificant modification of telescoping identity. We can show by induction that the error after $n>0$ steps is given by

$$
U^{n}(h)-u^{n}(h)=\sum_{j=0}^{n-1} U_{h}^{j}\left(U_{h}-u_{h}\right) u\left(t_{n-1-j}\right),
$$

where $U_{h}$ denotes the proposed scheme. As we proved in Proposition 3.5, $\left\|U_{h}^{j}\right\|$ is bounded by $K_{2}\left\|u_{0}\right\|$ and $u\left(t_{n-1-j}\right)$ is also bounded, say $\kappa$, because of the well-posedness of the solution. This yields

$$
\left\|U^{n}(h)-u^{n}(h)\right\| \leq \sum_{j=0}^{n-1} K_{2}\left\|\left(U_{h}-u_{h}\right) \kappa u_{0}\right\|
$$

Using Proposition 3.3, one can obtain

$$
\begin{aligned}
\left\|U^{n}(h)-u^{n}(h)\right\| & \leq \sum_{j=0}^{n-1} h^{3} G K_{2} \kappa\left\|u_{0}\right\| \\
& \leq h^{2} J_{1}
\end{aligned}
$$

Here $J_{1}=t_{\text {end }} \kappa K_{2}\left\|u_{0}\right\|$ where $G$ is described in Proposition 3.3 .

\section{Numerical Experiments}

In order to show the efficiency of the proposed method we will consider a few test problems. These include Duffing equation, Van-der Pol equation and Schrödinger equation.

\section{Duffing Equation}

Let us consider one dimensional unforced Duffing oscillator

$$
q^{\prime \prime}+\alpha q+\varepsilon q^{3}=0,
$$

where the initial conditions are $q(0)=1$ and $q^{\prime}(0)=0$. Here $\alpha$ controls the size of the stiffness and $\varepsilon$ controls the amount of nonlinearity in the restoring force. The analytic solution is given in [2] as

$$
q(t)=\cos t+\varepsilon\left[\frac{1}{32} \cos 3 t-\frac{1}{32} \cos t-\frac{3}{8} t \sin t\right], \quad \varepsilon \rightarrow 0^{+},
$$

where $\alpha=1$. 
First of all, we transform Eq. (52) into a system where $\alpha=1$ by redefining the variables as $q(t)=q_{1}(t)$ and $\dot{q}(t)=q_{2}(t)$, and $u(t)=\left(q_{1}(t), q_{2}(t)\right)^{T}$. The resulting system can be expressed in matrix form as follows:

$$
\begin{aligned}
\left(\begin{array}{l}
\dot{q}_{1} \\
\dot{q}_{2}
\end{array}\right) & =\left(\begin{array}{cc}
0 & 1 \\
-1-\varepsilon q_{1}^{2} & 0
\end{array}\right)\left(\begin{array}{l}
q_{1} \\
q_{2}
\end{array}\right) \\
& =\left(\begin{array}{cc}
0 & 1 \\
-1 & 0
\end{array}\right)\left(\begin{array}{l}
q_{1} \\
q_{2}
\end{array}\right)+\left(\begin{array}{cc}
0 & 0 \\
-\varepsilon q_{1}^{2} & 0
\end{array}\right)\left(\begin{array}{l}
q_{1} \\
q_{2}
\end{array}\right) .
\end{aligned}
$$

In order to compare the accuracy of different splitting methods given in Section 2, we solve Eq. (52) for $t_{\text {end }}=10$ and $\varepsilon=10^{-4}$ with different values of $\Delta t$.

In Table 1 , the convergence orders are presented in the discrete $L_{\infty}$-norm numerically. The obtained results are in perfect agreement with theoretical results. From Table 1, we also conclude that the new second order iterative splitting scheme is more accurate than not only Strang splitting but also symmetrically weighted splitting.

\begin{tabular}{rrrrrrr}
\hline & \multicolumn{2}{c}{ Proposed Method } & \multicolumn{2}{c}{ Strang Marchuk Splitting } & \multicolumn{2}{c}{ Symmetrically Weighted Splitting } \\
$\Delta t$ & Error & Order & Error & Order & Error & Order \\
\hline $1 / 8$ & $3.4097 \mathrm{e}-006$ & - & $7.2936 \mathrm{e}-006$ & - & $7.2704 \mathrm{e}-006$ & - \\
$1 / 16$ & $8.4748 \mathrm{e}-007$ & 2.0336 & $3.6175 \mathrm{e}-006$ & 1.0116 & $3.6150 \mathrm{e}-006$ & 1.0080 \\
$1 / 32$ & $2.08912 \mathrm{e}-007$ & 2.0203 & $1.7779 \mathrm{e}-006$ & 1.0248 & $1.7774 \mathrm{e}-006$ & 1.0242
\end{tabular}

Table 1: Comparison of errors for several values of $\mathrm{h}$ on the interval $[0,10]$ with various methods where $\varepsilon=10^{-4}$

\section{Van-der Pol Equation}

In the second test problem we consider the Van-der Pol equation which is one of the most potently studied systems in nonlinear dynamics. In our study, the second order autonomous Van-der Pol equation is discussed which is given as follows:

$$
\ddot{x}+\mu\left(x^{2}-1\right) \dot{x}+x=0, \quad x(0)=2, \quad \dot{x}(0)=0 .
$$

The parameter $\mu$ is a positive scalar denoting the nonlinearity and the strength of the damping. Considering $\dot{x}_{1}=x_{2}$ and $\dot{x}_{2}=-\mu\left(x_{1}^{2}-1\right) x_{2}-x_{1}$. After splitting, we obtain

$$
\left[\begin{array}{l}
\dot{x}_{1} \\
\dot{x}_{2}
\end{array}\right]=\left[\begin{array}{cc}
0 & 1 \\
-1 & 0
\end{array}\right]\left[\begin{array}{l}
x_{1} \\
x_{2}
\end{array}\right]+\left[\begin{array}{cc}
0 & 0 \\
0 & -\mu\left(x_{1}^{2}-1\right)
\end{array}\right]\left[\begin{array}{l}
x_{1} \\
x_{2}
\end{array}\right] \text {. }
$$

In order to see the effects of nonlinearity of the equation, we consider $\mu=10$. Since there is no exact solution for Eq. (55), therefore, the numerical results obtained from the proposed method are compared with the numerical results obtained using MATLAB routine ODE23. Fig. 1 shows that the proposed method is in a perfect agreement with the numerical solution of ODE23.

Furthermore, the phase diagrams of the solution obtained from both the proposed method and ODE23 are shown in Fig. 2, which are also in perfect agreement with each other.

We note that ODE23 uses an implicit method, whereas the proposed method is explicit. In spite of the fact that the new splitting method is explicit, it preserves the limit cycle of the given equation.

\section{Schrödinger Equation}

We consider the cubic nonlinear Schrödinger equation:

$$
\begin{aligned}
i \hbar \partial_{t} \psi & =\hat{H} \psi(x, t) \\
i \hbar \partial_{t} \psi & =\beta \partial_{x}^{2} \psi+\left(G(x)+\alpha|\psi|^{2}\right) \psi
\end{aligned}
$$




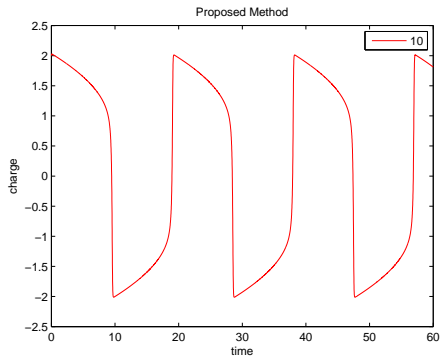

(a) The Proposed Method.

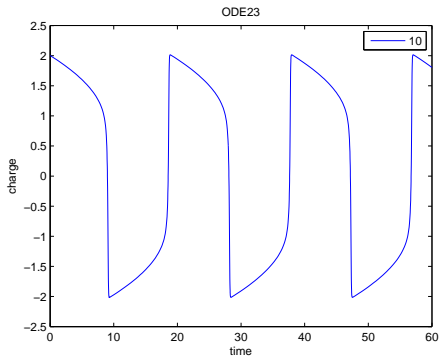

(b) ODE23s-code in MATLAB.

Figure 1: The numerical solutions obtained on $t \in[0,60]$ where $\mu=10$.

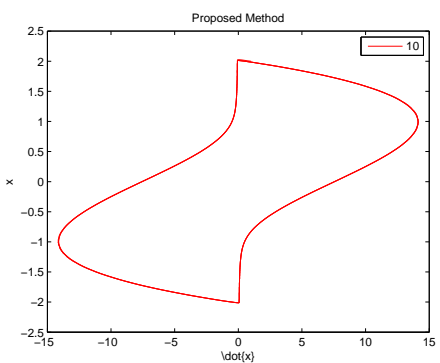

(a) The Proposed Method.

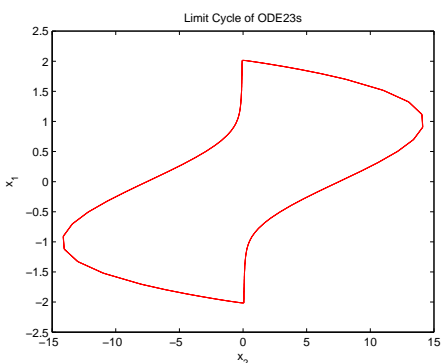

(b) ODE23s-code in MATLAB.

Figure 2: Trajectories of Equation (55) where $\mu=10$ and $t \in[0,60]$.

where $\psi(x, t)$ denotes the probability amplitude for the particle to be found at position $x$ at time $t$ and $\hbar$ is Planck constant. In order to transform the equation into system form, we make a change of variable $\psi$ as $\eta+i \xi$ in Eq. (58). This yields

$$
\hbar\left[\begin{array}{c}
\dot{\eta} \\
\dot{\xi}
\end{array}\right]=\left[\begin{array}{cc}
\mathbf{0} & \beta \partial_{x}^{2}+\left(G(x)+\alpha|\psi|^{2}\right) \\
-\beta \partial_{x}^{2}-\left(G(x)+\alpha|\psi|^{2}\right) & \mathbf{0}
\end{array}\right]\left[\begin{array}{l}
\eta \\
\xi
\end{array}\right] .
$$

Next, we split the system as $T+V(x, \psi)$ where

$$
T=\left[\begin{array}{cc}
\mathbf{0} & \beta \partial_{x}^{2} \\
-\beta \partial_{x}{ }^{2} & \mathbf{0}
\end{array}\right], V(x, \psi)=\left[\begin{array}{cc}
\mathbf{0} & \left(G(x)+\alpha|\psi|^{2}\right) \\
-\left(G(x)+\alpha|\psi|^{2}\right. & \mathbf{0}
\end{array}\right] .
$$

Here, $\partial_{x}^{2}$ denotes the spatial derivative and $\mathbf{0}$ denotes the zero matrix whose size equals to the size of $\partial_{x}{ }^{2}$. We use central difference to approximate the spatial derivative. Note that the matrices in Eq. (59) are skew-symmetric and therefore, the eigenvalues are purely imaginary. This guarantees that the solutions are bounded.

In this study, we consider $\hbar=1, \beta=-\frac{1}{2}, \alpha=30$ and $G(x)=\frac{1}{1+\sin ^{2} x}$, that is,

$$
i \partial_{t} \psi=-\frac{1}{2} \partial_{x}{ }^{2} \psi+\left(\frac{1}{1+\sin ^{2} x}+30|\psi|^{2}\right) \psi,
$$

with the Dirichlet boundary conditions

$$
\psi\left(x_{L}, t\right)=\psi\left(x_{R}, t\right)=0, t \in\left[0, t_{\text {end }}\right],
$$


where the initial condition is taken as $\psi_{0}(x)=\gamma \exp (\sin 2 x)$. Physically, the probability of any particle in Equation (60) is given by

$$
\int_{x_{L}}^{x_{R}}|\psi|^{2} d x \leq 1 .
$$

In order to see the validity of the solutions obtained from the proposed iterative splitting method, we need to set $\gamma$ as $\frac{1}{\left\|\psi_{0}\right\|}$ due to the following fact:

$$
\int_{x_{L}}^{x_{R}}\left|\psi_{0}\right|^{2} d x=1
$$

We solve Eq. (60) using the splitting process given in Eq. (59). The numerical solutions and the contour plots are shown for the proposed iterative splitting and the Strang splitting methods in Figs. 3 and 4, respectively.

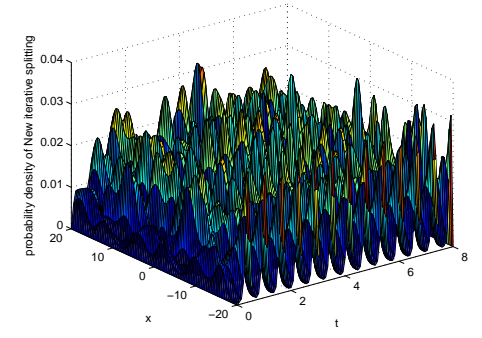

(a) Numerical Solution

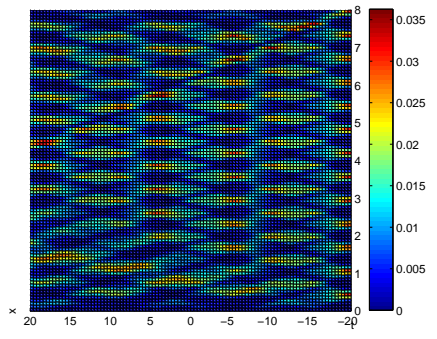

(b) Contour Plot.

Figure 3: Probability density of the particle in Equation (60) for the proposed method.

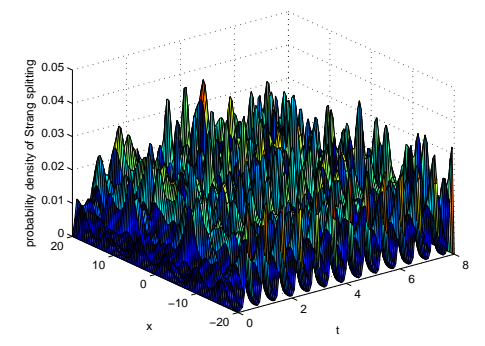

(a) Numerical Solution.

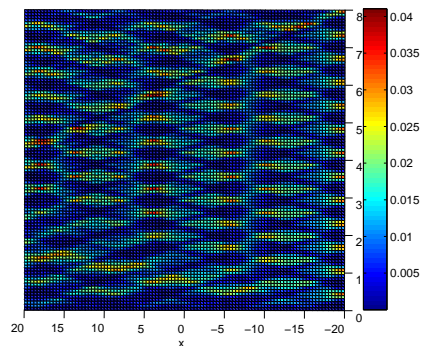

(b) Contour Plot.

Figure 4: Probability density of the particle in Equation (60) for Strang splitting method.

In these figure, we have assumed that the system is defined in the interval $x \in[-20,20]$, which is split into $N_{x}=100$ parts. We integrate the system using proposed iterative splitting method with the time-step size $\Delta t=0.08$ up to final time $t=8$. It can be observed that our method also preserves the probability density of the particle in (60).

In order to check the conservation of the mass, we have for the initial condition as

$$
\int_{-20}^{20}\left|\psi_{0}\right|^{2} d x=0.4000
$$


and at the end point, that is, for $t=8$, we have

$$
\int_{-20}^{20}\left|\psi_{P M}\right|^{2} d x=0.3788, \quad \int_{-20}^{20}\left|\psi_{S M}\right|^{2} d x=0.3780,
$$

for the numerical solutions of proposed iterative splitting method and Strang splitting denoted by $P M$ and $S M$, respectively. We observe that the difference between the initial mass and the final mass is negligible.

The CPU times for both the proposed method and Strang splitting method for different values of $N_{t}$ and $N_{x}$ are presented in Table 2. From the table it is obvious that the proposed method is more efficient than Strang splitting. Fig. 5 shows that the second order Runge-Kutta method of order 2 (RK2) does not

\begin{tabular}{lccccc}
\hline$N_{t}$ & $N_{x}$ & $\Delta t$ & $\Delta x$ & Proposed Method & Strang Marchuk Splitting \\
\hline 100 & 100 & 0.08 & 0.4 & 5.2188 & 6.1094 \\
200 & 100 & 0.04 & 0.4 & 9.4844 & 13.0625 \\
160 & 200 & 0.05 & 0.2 & 49.5938 & 75.1875 \\
200 & 200 & 0.04 & 0.2 & 56.7813 & 100.2969 \\
\hline
\end{tabular}

Table 2: CPU times for different $N_{t}$ and $N_{x}$

preserve the probability density of the particle in Eq. (60) with $t_{\text {end }}=5$, whereas the proposed method does, see Figure 3.

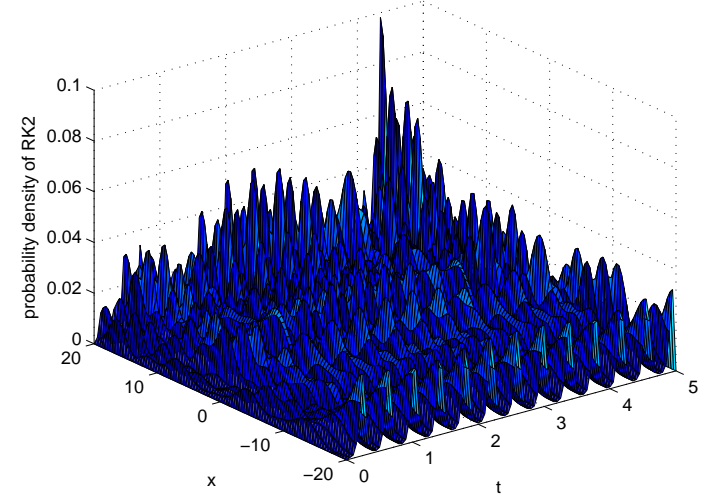

Figure 5: The numerical solutions obtained from the RK2 method for $t \in[0,5]$

\section{Conclusions and Discussions}

A new iterative method is proposed and analyzed for solving nonlinear oscillation problems. For the abstract analysis, $C_{0}$ semigroup property and the exponential integrators are used. In the case of Duffing equation, numerical results are confirmed with the theoretical results. Moreover, the proposed method has higher order accuracy than the other traditional splitting methods. In the case of Van der Pol equation, the proposed method is in perfect agreement with MATLAB routine ODE23. It is worth mentioning that ODE23 uses an implicit method whereas the proposed is explicit which is a clear advantage of the proposed method over ODE23. The proposed method also preserves the limit cycle of the Van der Pol equation. In the case of cubic nonlinear Schrödinger equation the proposed method preserves the probability density of the particle whereas the RK2 method does not. It is also shown that the proposed method is more efficient than the Strang splitting method in the case of Schrödinger equation. Although the set of test problems considered is a small sample of nonlinear oscillation equations, the method can be adapted in a simple way to solve other such problems numerically. 


\section{References}

[1] V.C. Aguilera-Navarro, G.A. Estévez and R. Guardiola, Variational and perturbative schemes for a spiked harmonic oscillator, J. Math. Phys. 31(99) (1990) http://dx.doi.org/10.1063/1.528832.

[2] C.M. Bender and S.A. Orszag, Advanced Mathematical Methods for Scientists and Engineers, Springer-Verlag, New York, 1999.

[3] S. Blanes and P.C. Moan, Splitting Methods for non-autonomous differential equations, J. Comput. Phys. 170 (2001) $205-230$.

[4] F. Casas and A. Iserles, Explicit Magnus expansions for nonlinear equations, J. Phys. A: Math. Gen. 39 (2006) 5445-5461.

[5] S.A. Chin and P. Anisimov, Gradient Symplectic Algorithms for Solving the Radial Schrödinger Equation, J. Chem. Phys. 124 054106 (2006)

[6] K.J. Engel and R. Nagel, A short course on operator semigroups, Universitext, Springer, New York, 2006.

[7] I. Faragó and J. Geiser, Iterative operator splitting methods for linear problems, International Journal of Computational Science and Engineering 3(4) (2007) 255-263.

[8] J. Geiser, Iterative Operator-Splitting Methods with higher order Time- Integration Methods and Applications for Parabolic Partial Differential Equations, Journal of Computational and Applied Mathematics 217 (2008) 227-242.

[9] J. Geiser, Decomposition methods for differential equations: Theory and application, CRC Press, Taylor and Francis Group, 2008.

[10] J. Geiser and L. Noack, Iterative operator-splitting methods for nonlinear differential equations and applications of deposition processes, Numerical Methods for Partial Differential Equations 27(5) (2011) 1026-1054.

[11] G. Goldstein and D. Baye, Sixth-order factorization of the evolution operator for time-dependent potentials, Phys. Rev E, 70 056703 (2004).

[12] M. Hochbruck and A. Ostermann, Exponential Integrator, Acta Numerica, Cambridge University Press (2010) 209-286.

[13] H. Holden, K. H. Karlsen and N. H. Risebro, Operator splitting methods for generalized Korteweg-de Vries equations, Journal of Computational Physics 153 (1999) 203-222.

[14] H. Holden, K. H. Karlsen, K. A. Lie and N. H. Risebro, Splitting Methods for Partial Differential Equations with Rough Solutions. Analysis and Matlab programs, European Mathematical Society, 2010.

[15] L. Lara, A numerical method for solving a system of nonautonomous linear ordinary differential equations, Applied Mathematics and Computation 170 (2005) 86-94.

[16] C. Lubich, On splitting methods for Schrödinger-Poisson and cubic nonlinear Schrödinger equations, Math. Comp. 77 (2008) 2141-2153.

[17] R. I. McLachlan and G. R. W. Quispel, Splitting Methods, Cambridge University Press (2002) 341-434.

[18] G. Tanoğlu and S. Korkut, The convergence of a new symmetric iterative splitting method for non-autonomous systems, International Journal of Computer Mathematics 89 (2012) 1837-1846. 